\title{
A Phase II Trial of the Multitargeted Tyrosine Kinase Inhibitor Lenvatinib (E7080) in Advanced Medullary Thyroid Cancer
}

\author{
Martin Schlumberger ${ }^{1}$, Barbara Jarzab², Maria E. Cabanillas ${ }^{3}$, Bruce Robinson ${ }^{4}$, \\ Furio Pacini ${ }^{5}$, Douglas W. Ball ${ }^{6}$, Judith McCaffrey ${ }^{7}$, Kate Newbold ${ }^{8}$, Roger Allison ${ }^{9}$, \\ Renato G. Martins ${ }^{10}$, Lisa F. Licitra"1, Manisha H. Shah ${ }^{12}$, Donald Bodenner ${ }^{13}$, Rossella Elisei ${ }^{14}$, \\ Lynn Burmeister ${ }^{15}$, Yasuhiro Funahashi ${ }^{16}$, Min Ren ${ }^{17}$, James P. O'Brien ${ }^{17}$, and \\ Steven I. Sherman ${ }^{3}$
}

\section{Abstract}

Purpose: Positive results of phase I studies evaluating lenvatinib in solid tumors, including thyroid cancer, prompted a phase II trial in advanced medullary thyroid carcinoma (MTC).

Experimental Design: Fifty-nine patients with unresectable progressive MTC per Response Evaluation Criteria In Solid Tumors (RECIST) v1.0 within the prior 12 months received lenvatinib (24-mg daily, 28-day cycles) until disease progression, unmanageable toxicity, withdrawal, or death. Prior anti-VEGFR therapy was permitted. The primary endpoint was objective response rate (ORR) by RECIST v1.0 and independent imaging review.

Results: Lenvatinib ORR was 36\% [95\% confidence interval (CI), 24\%-49\%]; all partial responses. ORR was comparable between patients with $(35 \%)$ or without (36\%) prior anti-VEGFR therapy. Disease control rate (DCR) was $80 \%$ (95\% CI, 67\%$89 \%) ; 44 \%$ had stable disease. Among responders, median time to response (TTR) was 3.5 months (95\% CI, 1.9-3.7). Median progression-free survival (PFS) was 9.0 months (95\% CI, 7.0-not evaluable). Common toxicity criteria grade $3 / 4$ treatment-emergent adverse events included diarrhea (14\%), hypertension (7\%), decreased appetite $(7 \%)$, fatigue, dysphagia, and increased alanine aminotransferase levels (5\% each). Ret proto-oncogene status did not correlate with outcomes. Low baseline levels of angiopoietin-2, hepatocyte growth factor, and IL8 were associated with tumor reduction and prolonged PFS. High baseline levels of VEGF, soluble VEGFR3, and platelet-derived growth factor BB, and low baseline levels of soluble Tie-2, were associated with tumor reduction.

Conclusions: Lenvatinib had a high ORR, high DCR, and a short TTR in patients with documented progressive MTC. Toxicities were managed with dose modifications and medications. Clin Cancer Res; 22(1); 44-53. (2015 AACR.

\section{Introduction}

Medullary thyroid carcinoma (MTC) is a rare neuroendocrine tumor arising from the parafollicular $\mathrm{C}$ cells in the thyroid gland. MTC accounts for about $4 \%$ of all thyroid cancers (1), whereas differentiated thyroid cancer (DTC), a tumor of epithelial origin, accounts for more than $90 \%$ (2).
The overall 10-year survival rate of patients with MTC ranges from $96 \%$ for patients with intrathyroid disease to $40 \%$ for patients with locally advanced disease or with distant metastases (3). Total thyroidectomy and neck lymph node dissection is considered the most effective therapeutic approach $(1,4,5)$, although patients with distant metastases may not fully benefit from extensive surgery (3). For these

Department of Nuclear Medicine and Endocrine Oncology, Institut Gustave-Roussy and University Paris-Sud, Villejuif, France. ${ }^{2}$ Department of Nuclear Medicine and Endocrine Oncology, Centrum Onkologii Instytut im. M. Sklodowskiei-Curie, Gliwice, Poland. ${ }^{3}$ Department of Endocrine Neoplasia and Hormonal Disorders, The University of Texas MD Anderson Cancer Center, Houston, Texas. ${ }^{4}$ Department of Endocrinology, Royal North Shore Hospital, Sydney, Australia. ${ }^{5}$ Department of Medical, Surgical and Neurological Sciences, Azienda Ospedaliera Universitaria Senese, Siena, Italy. ${ }^{6}$ Division of Endocrinology and Metabolism, Johns Hopkins Medical Institute, Baltimore, Maryland. ${ }^{7}$ Department of Interdisciplinary Oncology, H. Lee Moffitt Cancer Center and Research Institute, Tampa, Florida. ${ }^{8}$ NIHR Roya Marsden Hospital and Institute of Cancer Research BRC, London, UK ${ }^{9}$ Cancer Care Services, The Royal Brisbane and Women's Hospital, Herston, Australia. ${ }^{10}$ Division of Clinical Research, Seattle Cancer Care Alliance, Seattle, Washington. "Department of Medical Oncology, Fondazione IRCCS Istituto Nazionale dei Tumori, Milan, Italy. ${ }^{12}$ Department of Medical Oncology, The Ohio State University School of Medicine, Columbus, Ohio. ${ }^{13}$ Department of Otolaryngology-Head and Neck Surgery, University of Arkansas, Little Rock, Arkansas. ${ }^{14}$ Endo-

crine Unit, Department of Clinical and Experimental Medicine, University of Pisa, Pisa, Italy. ${ }^{15}$ Department of Medicine, University of Minnesota, Minneapolis, Minnesota. ${ }^{16}$ Eisai, Andover, Massachusetts. ${ }^{17} \mathrm{Eisa}$ Inc., Woodcliff Lake, New Jersey.

Note: Supplementary data for this article are available at Clinical Cancer Research Online (http://clincancerres.aacrjournals.org/).

Corresponding Author: Martin Schlumberger, Department of Nuclear Medicine and Endocrine Oncology, Centre de Référence Tumeurs Réfractaires de la Thyroïde, Gustave Roussy and University Paris-Sud, Villejuif, France. Phone: 33-14-2116095; Fax: 33-14-2116363; E-mail: martin.schlumberger@gustaveroussy.fr

doi: 10.1158/1078-0432.CCR-15-1127

(c)2015 American Association for Cancer Research 


\section{Translational Relevance}

Lenvatinib, an oral, multitargeted tyrosine kinase inhibitor of the VEGFR1-3, FGFR1-4, PDGFR $\alpha$, RET, and KIT signaling networks, demonstrated antitumor activity in phase I studies against several solid tumors, including in advanced medullary thyroid cancer (MTC). This phase II study demonstrated an objective response rate (ORR) for lenvatinib of $36 \%$ in patients with progressive MTC. The ORR was similar between patients with (35\%) and without prior anti-VEGF therapy (36\%). Treatment with lenvatinib was associated with a well-defined safety profile that was managed with dose modifications and medications. This study also explored potential biomarkers that may be indicative of a response to lenvatinib, with lower baseline levels of angiopoietin-2 associated with greater tumor reduction and prolonged progression-free survival. These findings support the continued investigation of lenvatinib for the management of advanced MTC.

patients with advanced disease, surgery is considered palliative and radiotherapy or chemotherapy is generally poorly effective (6).

Approximately $75 \%$ of MTC cases present in sporadic form and the remainder in a hereditary pattern $(7,8)$. Germline mutations in the RET proto-oncogene (RET; rearranged during transfection) occur in nearly all cases of hereditary MTC (9). Somatic RET mutations occur in approximately $40 \%$ of sporadic MTC cases and have been associated with aggressive disease and poor prognosis (10). In addition, RAS mutations may play a role in RET-negative MTC tumorigenesis (11). Aberrant RET- or RAS-mediated activation of signaling cascades (including the MAPK and phosphatidyl 4,5 bisphosphonate-3 kinase/alpha serine/threonine protein kinase pathways) is considered the major pathogenic event of MTC $(12,13)$.

MTC tumors are highly vascularized, with angiogenesis mediated principally by VEGFRs but also FGF and plateletderived growth factor (PDGF) and their receptors (14). Overexpression of VEGF and VEGFR2 has been observed in 50\% to $95 \%$ of MTC tumors and is associated with metastases (15-17). Currently, two multitargeted tyrosine kinase inhibitors (TKI)vandetanib and cabozantinib, each with activity against RET and VEGFR2- have been approved by the FDA and the European Medicines Agency for treatment of locally advanced or metastatic MTC on the basis of results from phase III trials demonstrating improved progression-free survival (PFS) over placebo $(18,19)$.

Lenvatinib is an oral, multitargeted TKI that inhibits VEGFR 13, FGFR 1-4, PDGFR $\alpha$, RET, and v-kit Hardy-Zuckerman 4 feline sarcoma viral oncogene homolog (KIT; refs. 20-23) that has recently been approved for the treatment of radioiodine-refractory DTC (RR-DTC) on the basis of the results of the SELECT phase III trial (24). Preclinical and phase I evidence of antitumor efficacy in a variety of solid tumor types, including thyroid cancer (2528), prompted the initiation of a phase II trial in advanced, unresectable progressive MTC and RR-DTC, stratified by histology (29). Here, we present results from the MTC component of the study.

\section{Materials and Methods}

\section{Study design and treatment}

This was a phase II, multicenter, open-label, single-arm clinical trial in patients with MTC and RR-DTC (Clinicaltrials.gov Trial Registration ID: NCT00784303). These are two distinct diseases with different prognoses; therefore, the study was designed with distinct entry criteria for each disease and to analyze and report the outcomes separately. The study was conducted at 30 sites in the United States, United Kingdom, Australia, France, Italy, and Poland, approved by the Institutional Review Board or Ethics Committee at each participating site and carried out in accordance with the Declaration of Helsinki. All patients provided written informed consent. Treatment consisted of once-daily oral lenvatinib administered at a starting dose of $24 \mathrm{mg}$ in 28-day treatment cycles for 8 cycles in the absence of disease progression, unmanageable toxicities, withdrawal, or death. Dose reductions occurred for grade $\geq 2$ adverse events (AE) except for hypertension, nausea, and vomiting, which were first managed by medical treatment. Once the dose was reduced, it could not be increased.

\section{Patients}

Patients were $\geq 18$ years of age with unresectable or metastatic histologically or cytologically confirmed MTC with at least one measurable lesion by Response Evaluation Criteria In Solid Tumors version 1.0 (RECIST v1.0; ref. 30) and either computed tomography (CT) or MRI scans. Patients also had documented disease progression within 12 months prior to study entry (as assessed by the investigator with CT or MRI scans per RECIST). Prior chemotherapy and anti-VEGFR treatments were allowed but must have been discontinued at least 30 days prior to study entry. Patients with significant cardiac, hematopoietic, hepatic, or renal dysfunction; metastases to the brain or leptomeningeal metastases; or use of anticoagulants were excluded.

\section{Tumor response}

The primary endpoint was objective response rate (ORR), per RECIST v1.0 criteria as assessed by independent imaging review (IIR). Secondary endpoints included PFS, overall survival (OS), disease control rate [DCR; defined as complete response (CR), partial response (PR), or stable disease (SD)], time to response (TTR) based on IIR, duration of response, and safety and tolerability. SD was defined as stable disease lasting $\geq 7$ weeks; durable SD was defined as SD for $\geq 23$ weeks.

\section{Patient monitoring and tumor assessment}

Physical examinations and laboratory evaluations were conducted on days 1, 8, 15, and 22 of cycle 1 ; on day 1 of cycle 2 ; and then on day 1 of every 28 -day cycle. Investigators were permitted to continue lenvatinib treatment until documented disease progression or unacceptable toxicity.

Within 4 weeks prior to initiation of treatment, tumor assessments using CT of the neck/chest/pelvis and MRI or CT of the abdomen were performed and assessed as the baseline tumor burden. Follow-up assessments in accordance with RECIST v1.0 were performed every other cycle or sooner if there was suspicion of progressive disease (PD). Tumor response was evaluated at the site, and if PR or CR was reported, the response was verified at least 4 weeks after first observed. The IIR of tumor response was used for the primary and secondary efficacy assessments. Designation of $\mathrm{SD}$ for best overall response (BOR) required at least one posttreatment assessment of SD at a minimum of 7 weeks after the first 
dose. At baseline [cycle 1 day 1 (C1D1)] and every 4 cycles, bone scans were performed.

Archival tumor tissues were available and obtained from 24 of 59 patients. Exploratory correlative analyses of tumor genetics, serum levels of 51 circulating cytokine and angiogenic factors (CAF), calcitonin (Ct), and carcinoembryonic antigen (CEA) with tumor responses to lenvatinib treatment were conducted. A total of 32 genes and 443 mutations were assessed using the Sequenom Mass ARRAY iPLEX OncoCarta v1.0 and v3.0 Platform (Sequenom, Inc.). A panel of 51 CAFs was assayed using ELISA and multiplex assays. Sample data acquisition and analysis were performed on either an ELISA plate reader using SoftmaxPro software (Molecular Devices) or the BioRad Bio-Plex System (Bio-Rad Laboratories) using Bio-Plex Manager 4.1 software for multiplex assays. CAFs for which $>20 \%$ of patients had out-ofrange measurements were not included in correlative analyses. $\mathrm{Ct}$ and CEA measurements were performed using chemiluminescence immunoassays on Immulite 2000 and Centaur instruments, respectively.

\section{Statistical analysis}

Sample size estimates were calculated on the basis of Simon optimal 2-stage design, assuming an expected ORR of $15 \%$ with lenvatinib compared with $2.5 \%$ based on historical controls, at $90 \%$ power and $\alpha$ of 0.5 . In this two-stage design, 16 patients were required in stage 1 ; if at least 1 confirmed CR or PR was observed by IIR, the study would continue to stage 2 to enroll a total of 52 patients. Otherwise, enrollment would be stopped for futility. However, because of rapid enrollment, all subjects were enrolled before the scheduled interim analysis (when the 16th evaluable patient completed 6 treatment cycles); nevertheless, because more than one confirmed responder was observed among the first 16 patients prior to the enrollment of additional patients, the criterion for moving to the second stage was already met. All patients received at least one lenvatinib dose and had at least one posttreatment safety assessment and were therefore included in both the intent-to-treat (ITT) and safety populations. The ITT population was used for the primary efficacy analyses. For the primary efficacy endpoint, ORR was calculated at the time of data cut offeither when all treated patients completed $\geq 8$ cycles or discontinued treatment prior to the eighth cycle. Two-sided 95\% confidence intervals (CI) for the ORR were calculated using Clopper and Pearson method. Descriptive statistics were used to summarize patient demographic data and safety parameters. PFS, estimation of PFS at 6 and 12 months, OS, and duration of response (for responders) were calculated using Kaplan-Meier productlimit estimates.

Mutation analyses were designed to test relationships between tumor mutation status and maximum change in tumor size, ORR, and PFS; for these analyses, the MannWhitney test, Fisher exact test, and log-rank test were applied, respectively. Posttreatment serum biomarker fold changes from baseline were analyzed by the Wilcoxon signed-rank test. Logtransformed values were used to test for associations between tumor responses and CAF levels. Circulating CAF levels were evaluated against maximum change in tumor size and PFS; for these analyses, the Spearman rank correlation test and the Cox proportional hazard model with Wald test $P$ values were applied, respectively. Kaplan-Meier curves for high and low CAF level groups using a median cutoff were drawn. To test for Ct or CEA changes and their associations with tumor shrinkage and PFS, the Spearman rank correlation test and univariate Cox proportional hazard models with Wald test $P$ values were applied, respectively. Unadjusted $P$ values and false discovery rates (FDR) for multiple comparisons are reported for the biomarker analyses. Results with $P<0.05$ were reported. Statistical analyses were performed using R version 2.15.0 or later ( $\mathrm{R}$ Foundation for Statistical Computing) with the following packages: survival and doMC (parallel computation).

\section{Results}

Patient demographics and baseline characteristics

Between October 2008 and August 2010, 162 patients with either DTC or MTC were screened and 117 were treated. For the MTC population $(n=59)$, the mean age was 52 years and the majority were male $(63 \%)$, White $(93 \%)$, had baseline Eastern Cooperative Oncology Group (ECOG) scores of $\leq 1$ (97\%), and New York Heart Association (NYHA) classification of 1 (85\%; Table 1). Furthermore, $44 \%$ had received prior anti-VEGFR therapy, $49 \%$ had received radiotherapy and 15\% had received conventional chemotherapy. The most common sites of metastases were liver (68\%), mediastinum (58\%), lung (53\%), and bone $(44 \%)$. Sixteen of the 24 analyzed patient tumors $(67 \%)$ were RET mutant-positive $\left(R E T^{\mathrm{M} 918 \mathrm{~T}}: n=14 ; \operatorname{RET}^{\mathrm{C} 634 \mathrm{R}}: n=2\right)$.

\section{Patient disposition}

Thirty (51\%) patients discontinued treatment for the following primary reasons: disease progression (25\%), AE (22\%), and patient choice $(3 \%)$.

\section{Tumor response}

After a minimum 8 months of follow-up, the ORR as assessed by IIR was 36\% (95\% CI, 24\%-49\%), with only PRs reported (Table 2). No obvious differences in ORR by age, gender, or prior anti-VEGFR therapy were observed. A BOR of SD was observed in $44 \%$ of patients and durable SD in $29 \%$ of patients. The DCR was $80 \%$ (95\% CI, 67\%-89\%). For patients who responded to treatment, the median TTR was 3.5 months (95\% CI, 1.9-3.7), and the median duration of response based on IIR assessments was not reached because of the high proportion of censored patients. A waterfall plot of the maximum tumor percentage change from baseline to postbaseline nadir is shown in Fig. 1A. The median PFS as assessed by IIR was 9.0 months [95\% CI, 7.0-not evaluable (NE); Fig. 1B]. The 6-month PFS rate was 67\% (95\% CI, 52\%$78 \%$ ) and the 12 -month PFS rate was $46 \%$ (95\% CI, 31\%-60\%). The overall median OS was 16.6 months (95\% CI, 16.4-NE; Supplementary Fig. S1).

\section{Tumor response based on prior anti-VEGFR therapy}

Tumor responses were similar regardless of prior history of antiVEGFR therapy. The ORR for patients who did $(n=26)$ or did not $(n=33)$ receive prior VEGFR therapy was 35\% (95\% CI, 17\%$56 \%$ ) and $36 \%$ (95\% CI, 20\%-55\%), respectively (all PRs). The median duration of response for patients who had received prior VEGFR treatment and responded to lenvatinib therapy $(n=9)$ was 5.7 months (95\% CI, 4.5-NE) and was not reached for lenvatinib responders without prior VEGFR treatment ( $n=$ 12). The median PFS was 7.3 months (95\% CI, 4.0-NE) in patients with prior VEGFR therapy and 12.9 months (95\% CI, 7.1-NE) for patients without prior VEGFR therapy. The median OS for patients with prior VEGF-targeted therapy was also 
Table 1. Patient demographics and baseline characteristics (ITT population)

\begin{tabular}{|c|c|}
\hline Category & Patients $(N=59)$ \\
\hline Mean age at study entry, y (min, max) & $51.6(22,74)$ \\
\hline \multicolumn{2}{|l|}{ Sex, $n(\%)$} \\
\hline Male & $37(63)$ \\
\hline Female & $22(37)$ \\
\hline \multicolumn{2}{|l|}{ Race/ethnicity, $n$ (\%) } \\
\hline White & $55(93)$ \\
\hline African American & $1(2)$ \\
\hline Other & $3(5)$ \\
\hline \multicolumn{2}{|l|}{ NYHA classification, $n$ (\%) } \\
\hline 1 & $50(85)$ \\
\hline 2 & $7(12)$ \\
\hline 3 or 4 & 0 \\
\hline Unknown & $2(3)$ \\
\hline \multicolumn{2}{|l|}{ ECOG performance status, $n$ (\%) } \\
\hline 0 & $32(54)$ \\
\hline 1 & $25(42)$ \\
\hline 2 & $2(3)$ \\
\hline $\begin{array}{l}\text { Patients with positive RET mutation } \\
\text { status, } n(\%)\end{array}$ & $16 / 24^{a}(67)$ \\
\hline \multicolumn{2}{|l|}{$\begin{array}{l}\text { Number of previous anticancer therapy } \\
\text { regimens, } n(\%)\end{array}$} \\
\hline 0 & $25(42)$ \\
\hline 1 & $22(37)$ \\
\hline 2 & $5(9)$ \\
\hline$\geq 3$ & $7(12)$ \\
\hline $\begin{array}{l}\text { Patients with previous conventional } \\
\text { chemotherapy, } n(\%)\end{array}$ & $9(15)$ \\
\hline Patients with previous radiotherapy, $n$ (\%) & $29(49)$ \\
\hline Patients with previous anti-VEGF therapy, $n$ (\%) & $26(44)$ \\
\hline Cabozantinib & $11(19)$ \\
\hline Sorafenib & $6(10)$ \\
\hline Vandetanib & $4(7)$ \\
\hline Motesanib & $3(5)$ \\
\hline Sunitinib & $3(5)$ \\
\hline Other & $3(5)$ \\
\hline \multicolumn{2}{|l|}{ Sites of metastasis at baseline ${ }^{b}$} \\
\hline Liver & $38(64)$ \\
\hline Bone & $19(32)$ \\
\hline Lung & $9(15)$ \\
\hline \multicolumn{2}{|l|}{ Symptoms at baseline } \\
\hline Diarrhea & $31(53)$ \\
\hline Musculoskeletal pain & $26(44)$ \\
\hline
\end{tabular}

a 24 Patients were evaluated for RET mutation status.

${ }^{\mathrm{b}}$ On the basis of study entry screening.

16.6 months (95\% CI, NE-NE), whereas median OS for patients without prior therapy was not reached at data cutoff.

\section{Pharmacogenomic and pharmacodynamics analyses}

An examination of genetic alterations in 24 archival patient tumor tissues yielded a total of 11 different mutations in 7 genes from 18 patient tumors. Of note, RET tumor mutation status did not show a statistically significant association with tumor shrinkage $(P=0.920)$, ORR $(P=1.000)$, or PFS $(P=0.313)$. An NRAS mutant-positive patient achieved SD and 2 patients with dual PIK3CA and RET ${ }^{\mathrm{M} 918 \mathrm{~T}}$ mutations had PRs.

After 8 days of treatment with lenvatinib, changes were observed in the levels of 13 of the 51 tested CAFs (Fig. 2). Notably, there were decreases in levels of 6 CAFs, including soluble VEGFR2 (sVEGFR2), sVEGFR3, and angiopoietin-2, and increases in levels of 7 CAFs: IFN $\gamma$-induced protein (IP)-10, VEGF, and stromal cellderived factor (SDF)-1a. Low baseline levels of angiopoeitin-2, Tie-2, hepatocyte growth factor (HGF), and IL8 and high baseline levels of VEGF, sVEGFR3, and the homodimer of PDGF-beta polypeptide (PDGF-BB) were associated with greater tumor shrinkage (Fig. 3A). Low baseline levels of angiopoietin-2, HGF, and IL8 were associated with prolonged PFS (Fig. 3B).

Serum Ct and CEA levels decreased upon lenvatinib treatment in almost all patients [Ct decreased by a median 0.49 -fold by cycle 2 day 1 (C2D1) in 47 of 52 patients; CEA decreased by a median 0.60 -fold by cycle 3 day 1 (C3D1) in 44 of 50 patients]. These changes were significantly correlated with ORR and prolonged PFS, respectively (C2D1 Ct, $P=0.012$ and $P=0.001$; HR, 1.72; 95\% CI, 1.25-2.38; C3D1 CEA, $P=0.032$ and $P=0.003$, HR, $2.06 ; 95 \% \mathrm{CI}, 1.28-3.32)$. When adjusted for multiple comparisons, these associations were no longer significant $(\mathrm{C} 2 \mathrm{D} 1 \mathrm{Ct}$, FDR $P=0.311$ for ORR and FDR $P=0.086$ for PFS; C3D1 CEA, FDR $P=0.327$ for ORR and FDR $P=0.114$ for PFS).

Treatment duration, safety, and tolerability

All patients experienced treatment-emergent AEs (TEAE). The most common TEAEs were diarrhea (75\%), proteinuria (59\%), fatigue (53\%), hypertension (51\%), decreased appetite (49\%), nausea $(48 \%)$, decreased weight $(42 \%)$, headache $(41 \%)$, vomiting (37\%), and cough (36\%; Table 3). Most hypertension and proteinuria events were grade 1 or 2 and managed with standard medical interventions. The median duration of treatment was 264 days (range, 13-547 days). At least 8 cycles of lenvatinib treatment were received by $64 \%$ of patients, with a mean dose intensity of $19.1 \mathrm{mg} / \mathrm{d}$. Dose reduction, interruption, or treatment withdrawal due to TEAEs was required in $59 \%, 75 \%$, and $24 \%$ of patients, respectively. Dose reductions to $20,14,10$, and $8 \mathrm{mg} / \mathrm{d}$ of lenvatinib occurred in $56 \%, 39 \%, 8 \%$, and $2 \%$ of patients, respectively. The median time to the first dose reduction was 2.2 months (range, 0.5-16.6). TEAEs that led to lenvatinib withdrawal and occurred in more than one patient were decreased appetite and decreased weight (3\% each). Withdrawal from treatment due to hypertension occurred in one patient (2\%).

Skin-related TEAEs included palmar-plantar erythrodysesthesia syndrome $(24 \%)$, rash $(22 \%)$, dry skin $(17 \%)$, alopecia and hyperkeratosis (12\% each), and skin exfoliation (10\%). One patient (2\%) experienced grade 1 folliculitis. Two patients (3\%) experienced grade 3 palmar-plantar erythrodysesthesia syndrome and one patient (2\%) experienced grade 4 exfoliative rash. The grade 4 exfoliative rash lasted for 8 days and was deemed unrelated to study treatment by the investigator; the study dose was therefore not changed, and the patient recovered.

Table 2. Tumor responses based on IIR assessments (ITT population)

\begin{tabular}{ll}
\hline Parameter, $\boldsymbol{n}(\%)$ & Overall $(\boldsymbol{N}=\mathbf{5 9})$ \\
\hline ORR $(C R+P R)$ & $21(36)$ \\
$95 \% \mathrm{Cl}$ & $24-49$ \\
Best overall tumor response $^{\mathrm{a}}$ & \\
CR & 0 \\
PR & $21(36)$ \\
SD $(\geq 7$ wks $)$ & $26(44)$ \\
Durable SD $\geq 23$ wks) & $17(29)$ \\
PD & $7(12)$ \\
NE & $2(3)$ \\
Unknown & $3(5)$ \\
DCR (CR + PR + SD) & $47(80)$ \\
$95 \%$ Cl & 67,89 \\
TTR, mo, median (95\% Cl) & $3.5(1.9-3.7)$ \\
Duration of response, mo, median $(95 \% \mathrm{Cl})$ & $\mathrm{NE}(5.7-\mathrm{NE})$ \\
\hline
\end{tabular}


A

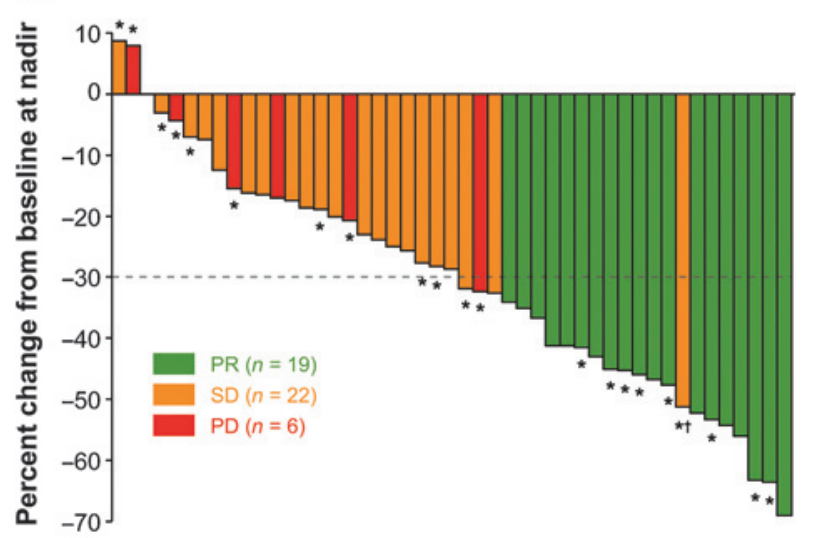

B

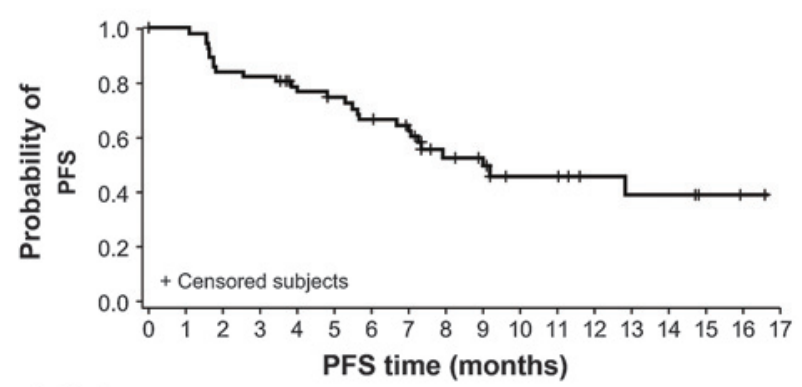

Subjects

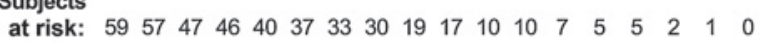

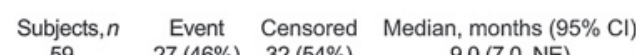

$\begin{array}{llll}59 & 27(46 \%) & 32(54 \%) & 9.0(7.0, \mathrm{NE})\end{array}$

Figure 1.

A, waterfall plot of percent change in summed longest diameter of target lesions from baseline by independent imaging review of the efficacy evaluable subjects per protocol $(n=50)$. No tumor change data were available for 3 patients with "Unknown" as their BOR. B, PFS by independent imaging review of the ITT population. *, patients previously treated with an antiVEGFR therapy; $\dagger$, although this patient had tumor shrinkage in the target lesion, the BOR of the patient was considered SD, as a baseline nontarget lesion became nonevaluable at a later assessment.

Common Toxicity Criteria (CTC) grade 3 TEAEs occurred in 36 patients (61\%). Grade 3 TEAEs that occurred in at least $5 \%$ of patients included diarrhea (14\%), hypertension $(7 \%)$, decreased appetite (7\%), fatigue (5\%), dysphagia (5\%), and increased levels of alanine aminotransferase (5\%). There were 5 grade 4 TEAEs that occurred in one patient each: increased levels of amylase, increased levels of lipase, exfoliative rash, accidental narcotic overdose, and pneumonia aspiration.

Serious AEs (SAE) occurred in $51 \%$ of patients and those that occurred in at least two patients included decreased appetite (5\%), pulmonary embolism, abdominal pain, pneumonia, lung infection, dehydration, and premature menopause (3.4\% each). SAEs led to dose interruption in $15.3 \%$, dose reduction in $8.5 \%$, and study drug withdrawal in $8.5 \%$ of patients. Four deaths occurred during treatment or within 30 days of the last lenvatinib dose. Of these, one death was due to clinical PD and 3 were due to AEs, including respiratory arrest (not otherwise specified), respiratory failure, and paraneoplastic syndromewith only the death by respiratory failure deemed treatmentrelated by the treating physician. An additional death occurred in a patient with tracheal-esophageal fistula that was study treatment-related; this event was recorded as an SAE, but not as a fatal AE.

\section{Discussion}

Distant metastases are the main cause of death in patients with advanced MTC with little evidence supporting the use of cytotoxic chemotherapy in these patients (14). Increased understanding of molecular mechanisms implicated in the pathogenesis and progression of MTC has prompted development of TKIs with activity against critical mediators of the relevant signaling pathways involved, including RET and VEGFR (6).

We evaluated oral lenvatinib (24 mg administered once-daily) for the treatment of unresectable or metastatic MTC and RECIST v1.0-documented disease progression at baseline in 59 patients, of which almost half of all patients had received prior anti-VEGFR treatment or had bone metastases. A confirmed ORR was observed in $36 \%$ of patients with only PRs reported. The median PFS was 9 months and the estimated PFS rate at 6 months was $67 \%$. In this study, although there was a numerical difference in median PFS between patients with and without prior VEGFtargeted therapy, tumor response was similar in both groups, confirming the lack of cross-resistance between TKIs previously suggested in a study of cabozantinib therapy in patients with prior VEGFR-targeted treatment (19).

Although results across different clinical trials are difficult to interpret, the tumor responses observed for lenvatinib in this trial are encouraging in the context of what has been reported for other TKIs. A phase II trial of vandetanib in patients with locally advanced or metastatic hereditary MTC showed a confirmed/unconfirmed PR rate of 30\% (31), and the subsequent phase III trial reported a $45 \%$ ORR in vandetanib-treated MTC patients, with a 6-month PFS rate of $83 \%(18)$. However, PD was not required to be present at study entry in either of these vandetanib trials, and the phase II trial was limited to patients with hereditary disease, both of which could have influenced the observed tumor response. Of note, a median PFS of 19 months was observed for placebo patients in the ZETA trial. In contrast, a phase III study of cabozantinib in unresectable locally advanced or metastatic MTC did require evidence of disease progression within 14 months of screening (19). Results showed statistically significant advantages in favor of cabozantinib over placebo in ORR $(28 \%$ vs. $0 \%)$ and the median PFS was 11.2 months in the cabozantinib arm and 4 months in the placebo arm. Therefore, despite the approval of both vandetanib and cabozantinib for the treatment of MTC, there is clearly still a need for effective TKI treatments in patients with progressive MTC.

Lenvatinib at the starting dose of $24 \mathrm{mg}$ once daily has a toxicity profile characterized by predominantly CTC grade $\leq 2$ TEAEs, including diarrhea, proteinuria, hypertension, fatigue, decreased appetite, nausea, decreased weight, vomiting, and abdominal pain. Twenty-two percent of patients withdrew from the study due to TEAEs. The AE profile of lenvatinib was generally consistent with anti-VEGFR treatment of advanced MTC (14). Most hypertension and proteinuria events were grade $\leq 2$ and most TEAEs were managed with standard medical care and dose interruption 
Figure 2.

Change in serum CAF levels during lenvatinib treatment. a, by ELISA; b, by multiplex array.

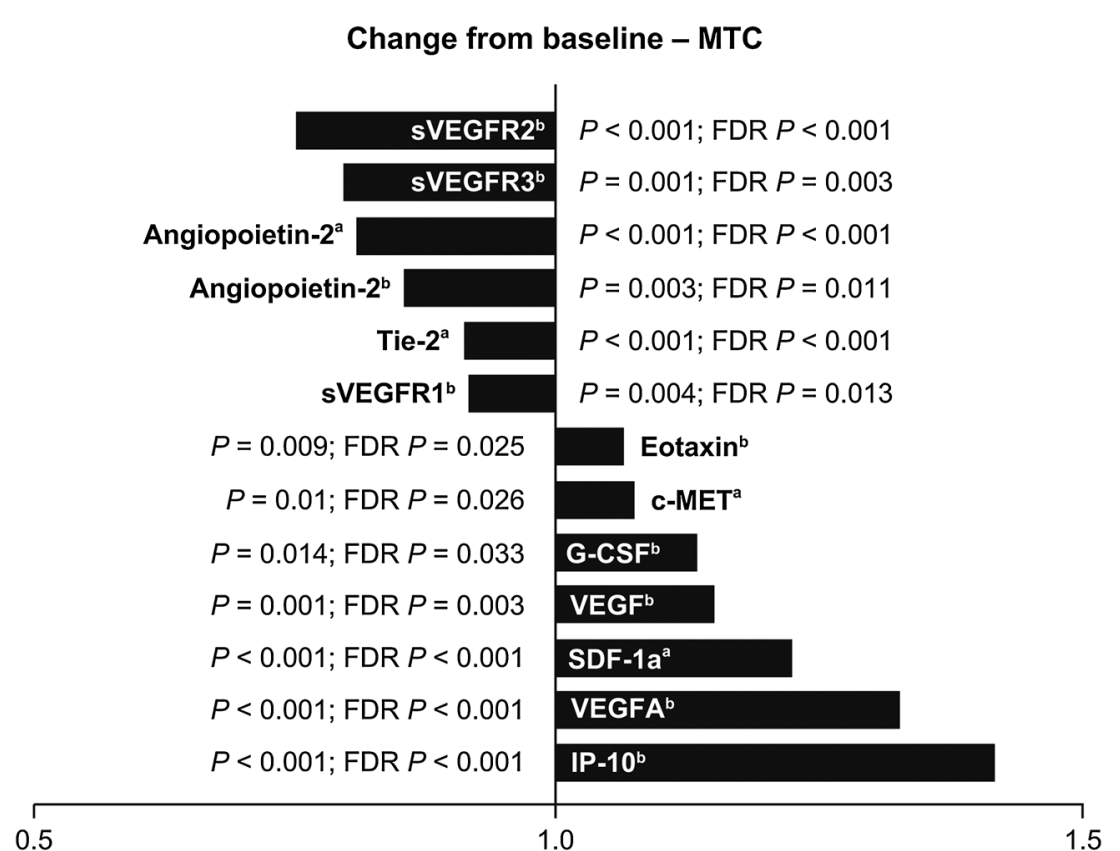

\begin{tabular}{|l|c|}
\hline Serum CAF & Median fold change \\
\hline sVEGFR2 $^{\mathrm{b}}$ & 0.753 \\
\hline sVEGFR3 $^{\mathrm{b}}$ & 0.800 \\
\hline Angiopoietin-2 $^{\mathrm{a}}$ & 0.810 \\
\hline Angiopoietin-2 $^{\mathrm{b}}$ & 0.855 \\
\hline Tie-2 $^{\mathrm{a}}$ & 0.916 \\
\hline sVEGFR1 $^{\mathrm{b}}$ & 0.918 \\
\hline Eotaxin $^{\mathrm{b}}$ & 1.067 \\
\hline c-MET $^{\mathrm{a}}$ & 1.074 \\
\hline G-CSF $^{\mathrm{b}}$ & 1.134 \\
\hline VEGF $^{\mathrm{b}}$ & 1.151 \\
\hline SDF-1 $^{\mathrm{a}}$ & 1.224 \\
\hline VEGFA $^{\mathrm{b}}$ & 1.329 \\
\hline IP-10 $^{\mathrm{b}}$ & 1.418 \\
\hline
\end{tabular}

or reduction when necessary. A high incidence of diarrhea was seen, although diarrhea is often also a complication of MTC. CTC grade 3 or 4 TEAEs, most of which were of grade 3 severity, were experienced by $70 \%$ of patients, most commonly diarrhea (12\%), hypertension $(7 \%)$, and decreased appetite (7\%). Fifty-one percent of patients had SAEs.

Of interest in this study was the generally low incidence of grade 3 skin toxicities. The incidence of palmar-plantar erythrodysesthesia syndrome (also known as hand-foot syndrome), was 24\%; grade 3 palmar-plantar erythrodysesthesia syndrome occurred in $3.4 \%$ of patients. The incidence of rash was $22 \%$ and one grade 4 exfoliative rash event occurred. In a phase II trial of sorafenib for metastatic MTC, the incidence of palmar-plantar erythrodysesthesia syndrome was $76 \%$ with grade $\geq 3$ events occurring in $14 \%$ of patients (32). In the same trial, the incidence of rash was $67 \%$ with no $\geq$ grade 3 rash events. In the ZETA trial,
$45 \%$ of vandetanib-treated patients experienced rash and $4 \%$ experienced grade $\geq 3$ rash events (18). In the present study, only one patient experienced grade 1 folliculitis. Folliculitis has been noted as a common $\mathrm{AE}$ identified in clinical studies with patients receiving vandetanib as treatment for MTC (33). Therefore, the use of lenvatinib may be associated with fewer skin toxicities, but this would need confirmation in placebo-controlled trials.

In this exploratory biomarker study of a limited number of patients, tumor response did not appear to correlate with RET mutation status. In addition, although RAS mutations are the second most important driver mutation in MTC, only a single NRAS-mutant tumor was identified in this study, possibly due to the limited number of tumors analyzed, as well as the method of genetic testing, which limited the range of mutations that could be identified. The associations found between changes in CAF levels 
Schlumberger et al.

and clinical outcomes of lenvatinib treatment suggest that antiangiogenic activity contributed to the observed antitumor activity in this study. This is consistent with results of a phase I clinical trial in metastatic MTC that showed that exposure to cabozantinib resulted in significant changes in the levels of placental growth factor, VEGF-A, and VEGFR2 (34). Correspondingly, the present study detected changes in the levels of sVEGFR2, sVEGFR3, and
VEGF-A in patient serum, as well as changes in levels of angiopoietin-2, sTie-2, SDF-1a, and IP-10 after 8 days of lenvatinib treatment.

We also observed that low baseline levels of angiopoietin-2, sTie-2, HGF, and IL8 were associated with greater tumor shrinkage; angiopoietin-2, HGF, and IL8 were additionally associated with prolonged PFS. HGF and IL8 are factors known to be
A

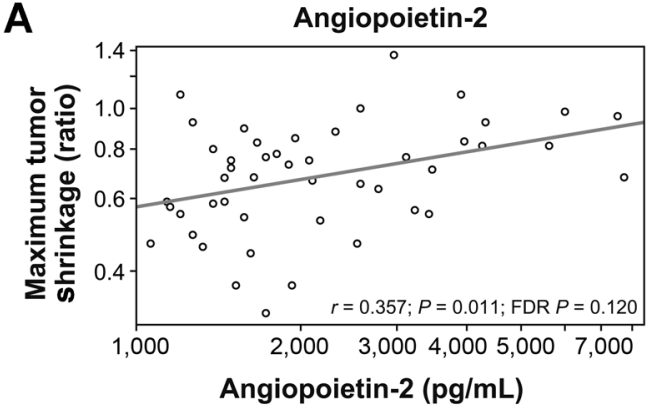

Tie-2

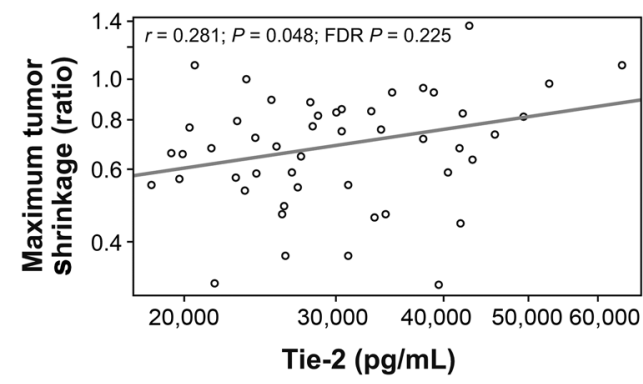

IL8

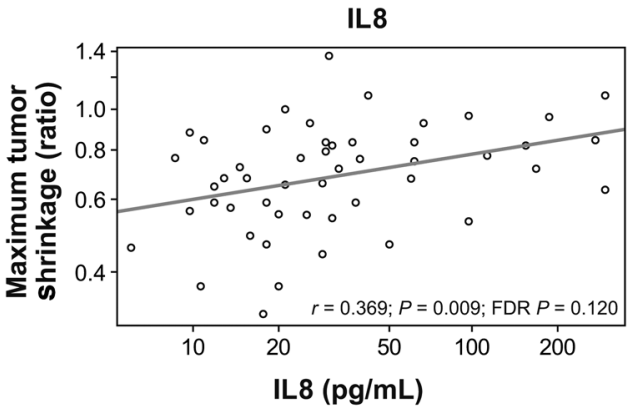

HGF

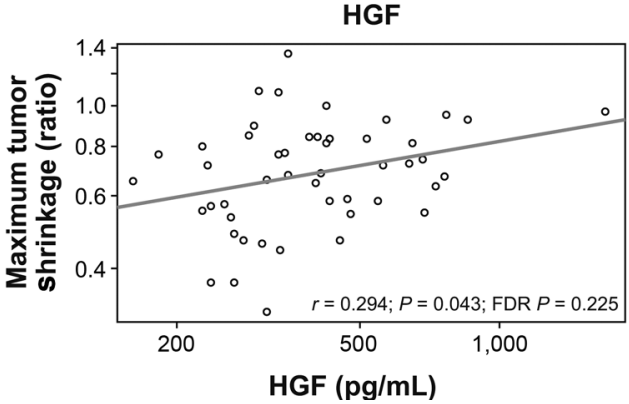

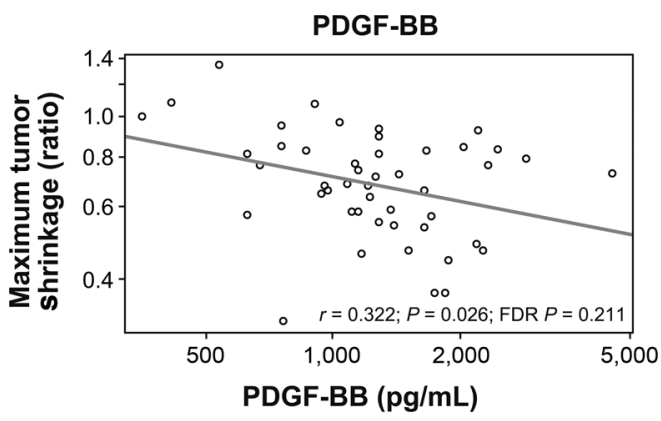

VEGF

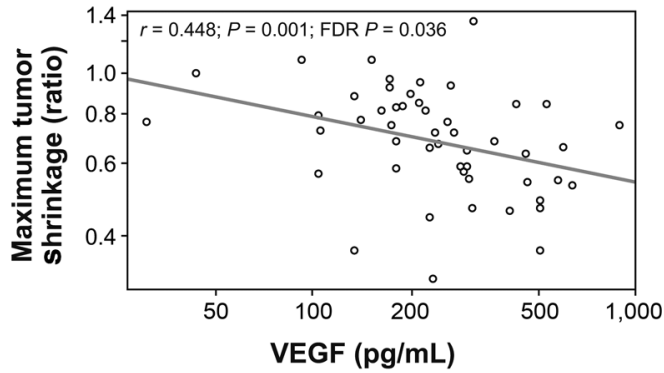

SVEGFR3

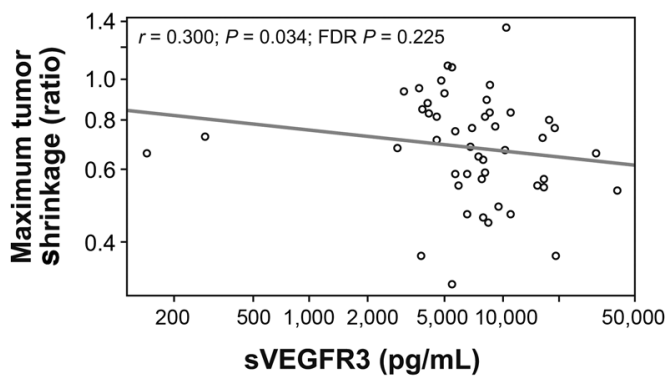

Figure 3.

Correlation of baseline cytokine and angiogenic factor levels with clinical outcome. A, correlation of baseline cytokine and angiogenic factor levels with maximum tumor shrinkage. B, Kaplan-Meier plots of PFS stratified by high and low baseline cytokine and angiogenic factor levels (median cutoff). (Continued on the following page.) 
B
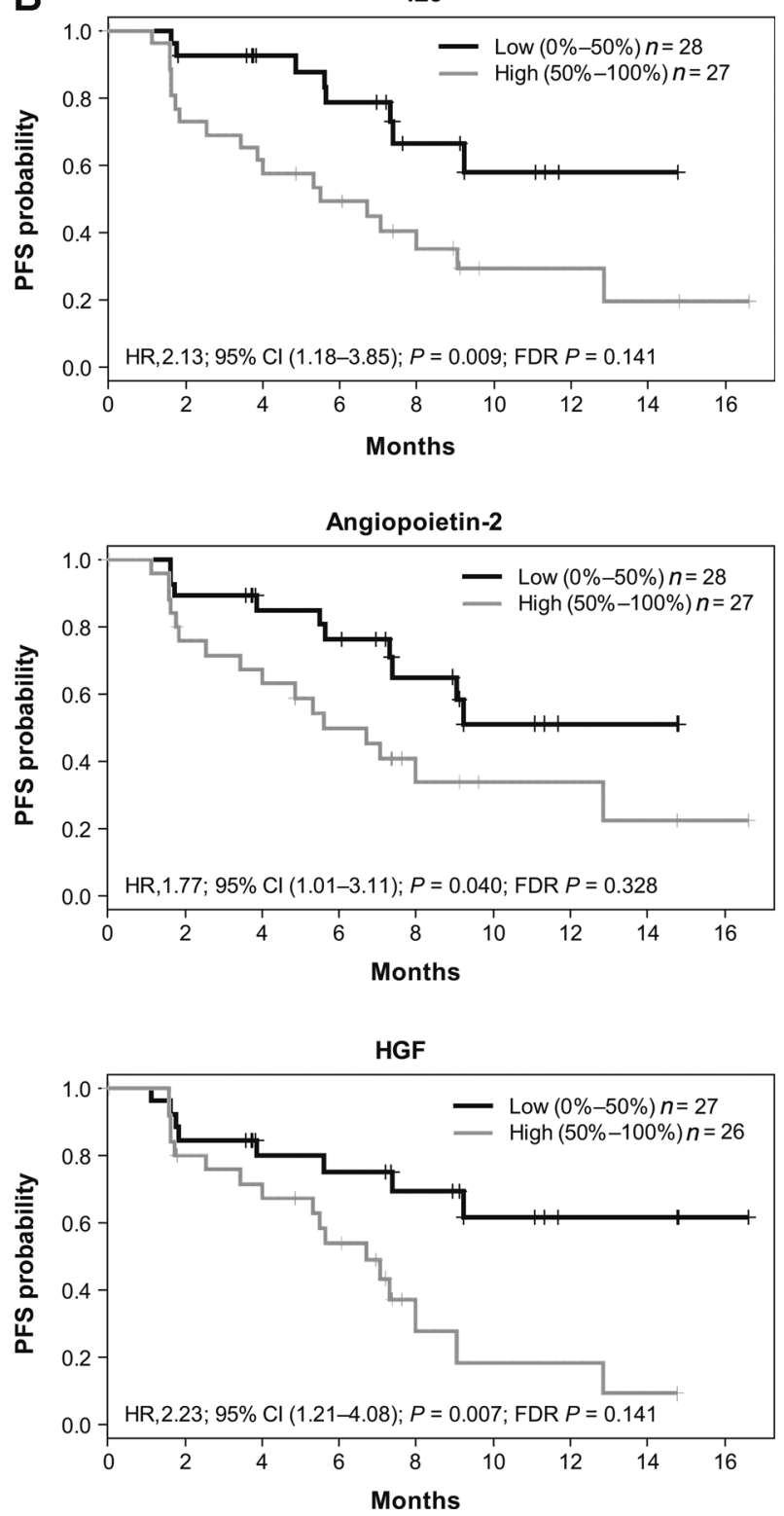

Figure 3.

(Continued)

associated with resistance to anti-VEGF therapy $(35,36)$. Our results suggest that angiopoietin-2/Tie-2 signaling may also contribute to VEGF or TKI treatment resistance (37); however, most of these markers lose statistical significance after adjustment for multiple analyses. Therefore, further study is needed to validate these proposed angiogenic biomarkers in appropriately powered and controlled clinical trials.

In conclusion, oral lenvatinib, dosed once daily at $24 \mathrm{mg}$, was associated with an ORR of $36 \%$, short TTR, prolonged duration of response, and a 6 -month PFS rate of $67 \%$. The observed toxicity profile was consistent with anti-VEGF treatment but with potentially greater incidence of weight loss and less clinically bothersome dermatological TEAEs. These results suggest that lenvatinib provides clinically meaningful tumor control with toxicities that
Table 3. TEAEs, all grades in $\geq 20 \%$ of patients

\begin{tabular}{lll}
\hline Event, & All grades & Grade $\mathbf{3 / 4}$ \\
$\boldsymbol{n}(\%)$ & $(\mathbf{N}=\mathbf{5 9 )}$ & $\mathbf{( N = \mathbf { 5 9 } )}$ \\
\hline Diarrhea & $44(75)$ & $8(14)$ \\
Proteinuria & $35(59)$ & $1(2)$ \\
Fatigue & $31(53)$ & $3(5)$ \\
Hypertension & $30(51)$ & $4(7)$ \\
Decreased appetite & $29(49)$ & $4(7)$ \\
Nausea & $28(48)$ & $1(2)$ \\
Decreased weight & $25(42)$ & $2(3)$ \\
Headache & $24(41)$ & $1(2)$ \\
Vomiting & $22(37)$ & 0 \\
Cough & $21(36)$ & 0 \\
Dysphonia & $19(32)$ & 0 \\
Arthralgia & $17(29)$ & $1(2)$ \\
Dyspnea & $16(27)$ & $1(2)$ \\
Abdominal pain upper & $15(25)$ & $1(2)$ \\
Abdominal pain & $15(25)$ & $1(2)$ \\
Pain in extremity & $15(25)$ & $2(3)$ \\
Constipation & $14(24)$ & $1(2)$ \\
Palmar-plantar erythrodysesthesia & $14(24)$ & $2(3)$ \\
$\quad$ syndrome & & \\
Musculoskeletal pain & $13(22)$ & 0 \\
Rash & $13(22)$ & 0 \\
Blood thyroid-stimulating & $12(20)$ & 0 \\
$\quad$ hormone level increased & $12(20)$ & 0 \\
Glossodynia & $12(20)$ & 0 \\
Myalgia & $12(20)$ & \\
Stomatitis & & \\
\hline & & \\
\hline
\end{tabular}

were managed by symptomatic treatments and dose modifications in this pretreated population of patients.

\section{Disclosure of Potential Conflicts of Interest}

M. Schlumberger is a consultant/advisory board member for AstraZeneca, Bayer, Eisai, and Exelixis. M.E. Cabanillas is a consultant/advisory board member for and reports receiving commercial research grants from Eisai. B. Robinson has ownership interest (including patents) in Mayne Pharma and is a consultant/ advisory board member for AstraZeneca, Bayer, and Eisai. D.W. Ball is a consultant/advisory board member for Eisai. K. Newbold reports receiving speakers bureau honoraria from and is a consultant/advisory board member for AstraZeneca, Eisai, and Genzyme. M.H. Shah reports receiving commercial research grants from Eisai and Exelixis. R. Elisei is a consultant/advisory board member for AstraZeneca, Bayer, Exelixis, and Genzyme. S.I. Sherman is a consultant/advisory board member for AstraZeneca, Bayer, Eisai, and Exelixis. No potential conflicts of interest were disclosed by the other authors.

\section{Authors' Contributions}

Conception and design: M. Schlumberger, B. Robinson, L.F. Licitra, Y. Funahashi, M. Ren, J.P. O'Brien, S.I. Sherman

Development of methodology: M. Schlumberger, L.F. Licitra, Y. Funahashi, M. Ren, S.I. Sherman

Acquisition of data (acquired and managed patients, provided facilities, etc.): M. Schlumberger, B. Jarzab, M.E. Cabanillas, B. Robinson, F. Pacini, D.W. Ball, J. McCaffrey, K. Newbold, R. Allison, R.G. Martins, L.F. Licitra, M.H. Shah, D. Bodenner, L. Burmeister, Y. Funahashi

Analysis and interpretation of data (e.g., statistical analysis, biostatistics, computational analysis): M. Schlumberger, M.E. Cabanillas, B. Robinson, L.F. Licitra, M.H. Shah, Y. Funahashi, M. Ren, S.I. Sherman

Writing, review, and/or revision of the manuscript: M. Schlumberger, B. Jarzab, M.E. Cabanillas, B. Robinson, F. Pacini, D.W. Ball, K. Newbold, R. Allison, R.G. Martins, L.F. Licitra, M.H. Shah, D. Bodenner, R. Elisei, Y. Funahashi, M. Ren, J.P. O'Brien, S.I. Sherman

Administrative, technical, or material support (i.e., reporting or organizing data, constructing databases): M. Schlumberger, L.F. Licitra, M.H. Shah Study supervision: M. Schlumberger, L.F. Licitra, R. Elisei, J.P. O'Brien Other (biomarker research): Y. Funahashi 


\section{Acknowledgments}

The authors thank Mark Matijevic and Tadashi Kadowaki for their assistance in the biomarker analyses. Medical editorial writing assistance was provided by Phase Five Communications Inc., and Oxford PharmaGenesis, Inc. The authors retained full editorial control over the article.

\section{Grant Support}

This work was supported by Eisai Inc.

\section{References}

1. Kloos RT, Eng C, Evans DB, Francis GL, Gagel RF, Gharib H, et al. Medullary thyroid cancer: management guidelines of the American Thyroid Association. Thyroid 2009;19:565-612.

2. Sherman SI. Thyroid carcinoma. Lancet 2003;361:501-11.

3. Roman S, Lin R, Sosa JA. Prognosis of medullary thyroid carcinoma: demographic, clinical, and pathologic predictors of survival in 1252 cases. Cancer 2006; 107:2134-42.

4. Scollo C, Baudin E, Travagli JP, Caillou B, Bellon N, Leboulleux S, et al. Rationale for central and bilateral lymph node dissection in sporadic and hereditary medullary thyroid cancer. J Clin Endocrinol Metab 2003;88: 2070-5.

5. Sippel RS, Kunnimalaiyaan M, Chen H. Current management of medullary thyroid cancer. Oncologist 2008;13:539-47.

6. Antonelli A, Fallahi P, Ferrari SM, Mancusi C, Colaci M, Santarpia L, et al. RET TKI: potential role in thyroid cancers. Curr Oncol Rep 2012; 14:97-104

7. Lakhani VT, You YN, Wells SA. The multiple endocrine neoplasia syndromes. Annu Rev Med 2007;58:253-65.

8. Leboulleux S, Baudin E, Travagli JP, Schlumberger M. Medullary thyroid carcinoma. Clin Endocrinol (Oxf) 2004;61:299-310.

9. Romei C, Mariotti S, Fugazzola L, Taccaliti A, Pacini F, Opocher G, et al Multiple endocrine neoplasia type 2 syndromes (MEN 2): results from the ItaMEN network analysis on the prevalence of different genotypes and phenotypes. Eur J Endocrinol 2010;163:301-8.

10. Elisei R, Cosci B, Romei C, Bottici V, Renzini G, Molinaro E, et al. Prognostic significance of somatic RET oncogene mutations in sporadic medullary thyroid cancer: a 10-year follow-up study. J Clin Endocrinol Metab 2008; 93:682-7.

11. Moura MM, Cavaco BM, Pinto AE, Leite V. High prevalence of RAS mutations in RET-negative sporadic medullary thyroid carcinomas. J Clin Endocrinol Metab 2011;96:E863-8.

12. Tamburrino A, Molinolo AA, Salerno P, Chernock RD, Raffeld M, Xi L, et al Activation of the mTOR pathway in primary medullary thyroid carcinoma and lymph node metastases. Clin Cancer Res 2012;18:3532-40.

13. Blaugrund JE, Johns MM Jr, Eby YJ, Ball DW, Baylin SB, Hruban RH, et al. RET proto-oncogene mutations in inherited and sporadic medullary thyroid cancer. Hum Mol Genet 1994;3:1895-7.

14. Schlumberger M, Massicotte MH, Nascimento CL, Chougnet C, Baudin E, Leboulleux S. Kinase inhibitors for advanced medullary thyroid carcinoma. Clinics (Sao Paulo) 2012;67:125-9.

15. Bunone G, Vigneri P, Mariani L, Butó S, Collini P, Pilotti S, et al. Expression of angiogenesis stimulators and inhibitors in human thyroid tumors and correlation with clinical pathological features. Am J Pathol 1999;155: 1967-76.

16. Capp C, Wajner SM, Siqueira DR, Brasil BA, Meurer L, Maia AL Increased expression of vascular endothelial growth factor and its receptors, VEGFR-1 and VEGFR-2, in medullary thyroid carcinoma. Thyroid 2010;20:863-71.

17. Rodríguez-Antona C, Pallares J, Montero-Conde C, Inglada-Pérez L, Castelblanco E, Landa I, et al. Overexpression and activation of EGFR and VEGFR2 in medullary thyroid carcinomas is related to metastasis. Endocr Relat Cancer 2010;17:7-16.

18. Wells SA Jr, Robinson BG, Gagel RF, Dralle H, Fagin JA, Santoro M, et al. Vandetanib in patients with locally advanced or metastatic medullary thyroid cancer: a randomized, double-blind phase III trial. J Clin Oncol 2012;30:134-41.

19. Elisei R, Schlumberger MJ, Müller SP, Schöffski P, Brose MS, Shah MH, et al. Cabozantinib in progressive medullary thyroid cancer. J Clin Oncol 2013;31:3639-46
The costs of publication of this article were defrayed in part by the payment of page charges. This article must therefore be hereby marked advertisement in accordance with 18 U.S.C. Section 1734 solely to indicate this fact.

Received May 15, 2015; revised July 21, 2015; accepted August 16, 2015; published OnlineFirst August 26, 2015.

20. Matsui J, Yamamoto Y, Funahashi Y, Tsuruoka A, Watanabe T, Wakabayashi T, et al. E7080, a novel inhibitor that targets multiple kinases, has potent antitumor activities against stem cell factor producing human small cell lung cancer H146, based on angiogenesis inhibition. Int J Cancer 2008;122:664-71.

21. Matsui J, Funahashi Y, Uenaka T, Watanabe T, Tsuruoka A, Asada M. Multikinase inhibitor E7080 suppresses lymph node and lung metastases of human mammary breast tumor MDA-MB-231 via inhibition of vascular endothelial growth factor-receptor (VEGF-R) 2 and VEGF-R3 kinase. Clin Cancer Res 2008;14:5459-65.

22. Okamoto K, Kodama K, Takase K, Sugi NH, Yamamoto Y, Iwata M, et al. Antitumor activities of the targeted multi-tyrosine kinase inhibitor lenvatinib (E7080) against RET gene fusion-driven tumor models. Cancer Lett 2013;340:97-103.

23. Yamamoto Y, Matsui J, Matsushima T, Obaishi H, Miyazaki K, Nakamura $\mathrm{K}$, et al. Lenvatinib, an angiogenesis inhibitor targeting VEGFR/ FGFR, shows broad antitumor activity in human tumor xenograft models associated with microvessel density and pericyte coverage. Vasc Cell 2014;6:18

24. Schlumberger M, Tahara M, Wirth LJ, Robinson B, Brose MS, Elisei R, et al. Lenvatinib versus placebo in radioiodine-refractory thyroid cancer. $\mathrm{N}$ Engl J Med 2015;372:621-30.

25. Matsui J, Minoshima Y, Tsuruoka A, Funahashi Y. Multi-targeted kinase inhibitor E7080 showed anti-tumor activity against medullary thyroid carcinoma and squamous thyroid carcinoma cell line based on RET and VEGFR2 tyrosine kinase inhibition [abstract]. Cancer Res 2010;70: Abstract 3614

26. Glen H, Mason S, Patel H, Macleod K, Brunton VG. E7080, a multi-targeted tyrosine kinase inhibitor suppresses tumor cell migration and invasion. BMC Cancer 2011;11:309.

27. Boss DS, Glen H, Beijnen JH, Keesen M, Morrison R, Tait B, et al. A phase I study of E7080, a multitargeted tyrosine kinase inhibitor, in patients with advanced solid tumours. Br J Cancer 2012;106:1598-604.

28. Tohyama O, Matsui J, Kodama K, Hata-Sugi N, Kimura T, Okamoto $\mathrm{K}$, et al. Antitumor activity of lenvatinib (e7080): an angiogenesis inhibitor that targets multiple receptor tyrosine kinases in preclinical human thyroid cancer models. J Thyroid Res 2014;2014: 638747.

29. Cabanillas ME, Schlumberger M, Jarzab B, Martins RG, Pacini F, Robinson $\mathrm{B}$, et al. A phase 2 trial of lenvatinib (E7080) in advanced, progressive, radioiodine-refractory, differentiated thyroid cancer: a clinical outcomes and biomarker assessment. Cancer 2015;121:2749-56.

30. Therasse P, Arbuck SG, Eisenhauer EA, Wanders J, Kaplan RS, Rubinstein L, et al. New guidelines to evaluate the response to treatment in solid tumors. European Organization for Research and Treatment of Cancer, National Cancer Institute of the United States, National Cancer Institute of Canada. J Natl Cancer Inst 2000;92:205-16.

31. Wells SA Jr, Gosnell JE, Gagel RF, Moley J, Pfister D, Sosa JA, et al. Vandetanib for the treatment of patients with locally advanced or metastatic hereditary medullary thyroid cancer. J Clin Oncol 2010;28 767-72.

32. Lam ET, Ringel MD, Kloos RT, Prior TW, Knopp MV, Liang J, et al. Phase II clinical trial of sorafenib in metastatic medullary thyroid cancer. J Clin Oncol 2010;28:2323-30.

33. European Medicines Agency. Committee for medicinal products for human use (CHMP). Caprelsa (vandetanib). Procedure no. EMEA/H/C/002315// 0000. 2011 [cited 2015 May 11]. Available from: http://www.ema.europa.eu/ docs/en_GB/document_library/EPAR__Public_assessment_report/human/ 002315/WC500123603.pdf. 
34. Kurzrock R, Sherman SI, Ball DW, Forastiere AA, Cohen RB, Mehra R, et al. Activity of XL184 (Cabozantinib), an oral tyrosine kinase inhibitor, in patients with medullary thyroid cancer. J Clin Oncol 2011;29:2660-6.

35. Huang D, Ding Y, Zhou M, Rini BI, Petillo D, Qian CN, et al. Interleukin- 8 mediates resistance to antiangiogenic agent sunitinib in renal cell carcinoma. Cancer Res 2010;70:1063-71.
36. Shojaei F, Lee JH, Simmons BH, Wong A, Esparza CO, Plumlee PA, et al. $\mathrm{HGF} / \mathrm{c}-\mathrm{Met}$ acts as an alternative angiogenic pathway in sunitinib-resistan tumors. Cancer Res 2010;70:10090-100.

37. Rigamonti N, Kadioglu E, Keklikoglou I, Wyser Rmili C, Leow CC, De Palma M. Role of angiopoietin-2 in adaptive tumor resistance to VEGF signaling blockade. Cell Rep 2014;8:696-706. 


\section{Clinical Cancer Research}

\section{A Phase II Trial of the Multitargeted Tyrosine Kinase Inhibitor Lenvatinib (E7080) in Advanced Medullary Thyroid Cancer}

Martin Schlumberger, Barbara Jarzab, Maria E. Cabanillas, et al.

Clin Cancer Res 2016;22:44-53. Published OnlineFirst August 26, 2015.

Updated version Access the most recent version of this article at: doi:10.1158/1078-0432.CCR-15-1127

Supplementary Access the most recent supplemental material at:

Material http://clincancerres.aacrjournals.org/content/suppl/2015/08/25/1078-0432.CCR-15-1127.DC1

Cited articles This article cites 36 articles, 14 of which you can access for free at:

http://clincancerres.aacrjournals.org/content/22/1/44.full\#ref-list-1

Citing articles This article has been cited by 3 HighWire-hosted articles. Access the articles at:

http://clincancerres.aacrjournals.org/content/22/1/44.full\#related-urls

E-mail alerts Sign up to receive free email-alerts related to this article or journal.

Reprints and To order reprints of this article or to subscribe to the journal, contact the AACR Publications Department at Subscriptions pubs@aacr.org.

Permissions To request permission to re-use all or part of this article, contact the AACR Publications Department at permissions@aacr.org. 